\title{
Sudden death and birth of entanglement in photonic crystals
}

\author{
M. Al-Amri, ${ }^{1,2,3, *}$ Gao-xiang Li, ${ }^{3, \dagger}$ Rong Tan, ${ }^{3}$ and M. Suhail Zubairy ${ }^{2,4}$ \\ ${ }^{1}$ The National Center for Mathematics and Physics, KACST, P.O. Box 6086, Riyadh 11442, Saudi Arabia \\ ${ }^{2}$ Max-Planck-Institut für Kernphysik, Saupfercheckweg 1, 69117 Heidelberg, Germany \\ ${ }^{3}$ Department of Physics, Huazhong Normal University, Wuhan 430079, China \\ ${ }^{4}$ Department of Physics and Institute of Quantum Studies, Texas A\&M University, College Station, Texas 77843-4242, USA
}

(Received 18 January 2009; published 11 August 2009)

\begin{abstract}
We explore the entanglement dynamics of two qubits embedded in one-dimensional (1D) and threedimensional (3D) photonic crystals. Our 1D results show sudden death and sudden birth of the entanglement, restricted by the initial coherence and the population in the excited state as well as the detuning. The results for the $3 \mathrm{D}$ crystal show entanglement death but no birth. We also discuss the entanglement of $N$ qubits in the aforementioned photonic crystals.
\end{abstract}

DOI: $10.1103 /$ PhysRevA.80.022314

PACS number(s): 03.67.Bg, 03.67.Mn, 03.65.Yz, 03.65.Ud

\section{INTRODUCTION}

Quantum entanglement lies at the heart of quantum world mystery and is the key for potential applications in quantum information $[1,2]$. Since quantum systems are easily affected by decoherence via an interaction with the environment, one of the big challenges is to find ways to maintain the entanglement for a longer time [3]. In order to achieve this, a good understanding of the decoherence mechanism is desirable. Yu and Eberly [4-6] showed that dynamical behavior of the entanglement between two qubits interacting independently with quantum or classical noise is different from the behavior of the local coherence. Instead of the exponential decay in time of the local decoherence, quantum entanglement may disappear within a finite time in the dynamical evolution. This phenomenon is called "entanglement sudden death" (ESD) [4]. Further investigations of ESD in different systems have been made [7-10]. ESD of two qubits system under the influence of independent environment has been carried out experimentally $[11,12]$. The concept of creation or revivals of entanglement has also been investigated [13-15].

Most of these studies are related to the entanglement dynamics of a bipartite system. A question of interest is how the phenomena of entanglement sudden death and birth happen in multipartite system. This is the subject of the present paper. A particularly interesting multipartite entangled state is the Greenberger-Horne-Zellinger (GHZ) type state which has interesting applications in secure quantum communication. These states have been realized experimentally for large number of particles. For example, five-photon GHZ entangled state [16] and ten-party GHZ state have been produced [17]. A GHZ state has also reported for six trapped ions [18]. However, it is well known that scaling multipartite entangled states up to many constituents is haunted by the decoherence processes. Therefore it is important to study the dynamical behavior of the multipartite GHZ state in the presence of decoherence. For example, Dür and Briegel [19] found that, for the GHZ states, the lifetime of true $N$-party

\footnotetext{
*mdalamri@kacst.edu.sa

†gaox@phy.ccnu.edu.cn
}

entanglement decreases with the size of the system and the effective number of entangled subsystem decreases with time. Aolita et al. [20] predicted that the disentanglement time of the GHZ-type states grows with the number of parties $N$, and the residual entanglement is reduced to an arbitrarily small value on a time scale that decreases with increasing $N$. All these studies are concerned for the cases of GHZ states in a Markovian (memoryless) environment.

In this paper, we present a study for the evolution of quantum entanglement between multiple qubits embedded in photonic band gap materials. We investigate the effect of decoherence on entanglement in one-dimensional (1D) and threedimensional (3D) photonic band gap materials. The properties of the individual qubits, their mutual interactions and the coherence dynamics are liable to modifications. The decoherence dynamics of two entangled qubits in a photonics crystal, such as entanglement trapping, is known to be different from that of two qubits in free vacuum $[21,22]$. The key feature here is the density of state (DOS) which should have an impact on the entanglement behavior [23-25].

The paper is organized as follows. In Sec. II, we present the single qubit spontaneous emission calculation in $1 \mathrm{D}$ and 3D photonic crystals. In the following subsections, we investigate the density matrix of two qubits that are placed separately in two photonic crystals. We extend our calculation to $N$ qubits separately in $N$ crystals with the initials preparation in GHZ state. In Sec. III we illustrate the time evolution of the entanglement for the qubits that are located individually in photonic crystals. Here, we figure out the situations where death and birth of the entanglement can take place. We summarize our results in Sec. IV.

\section{SPONTANEOUS EMISSION INSIDE PHOTONIC CRYSTALS}

We consider a system of $N$ qubits represented by twolevel atoms embedded inside $N$ photonic crystals such that each photonic crystal has only one qubit embedded in it. Since each qubit is located in a separate photonic crystal, it evolves independently via interaction with reservoirs.

\section{A. Single qubit spontaneous emission}

Following [23,26], we consider a two-level atom embedded in a photonic crystal coupled to the radiation field. The 
transition frequency $\omega_{a}$ between the excited and the ground atomic states $|e\rangle$ and $|g\rangle$, respectively, is resonant with the radiation field. The Hamiltonian for the system is [27]

$$
H=\hbar \omega_{a}|e\rangle\langle e|+\sum_{k} \hbar \omega_{k} a_{k}^{\dagger} a_{k}+i \hbar \sum_{k} g_{k}\left(a_{k}^{\dagger}|g\rangle\left\langle e\left|-a_{k}\right| e\right\rangle\langle g|\right),
$$

where $a_{k}^{\dagger}$ and $a_{k}$ are the radiation field creation and annihilation operators for the $k$ th radiation mode with frequency $\omega_{k}$. The parameter $g_{k}=\omega_{a} d\left(2 \epsilon_{0} \omega_{k} V\right)^{-1 / 2} \mathbf{e}_{k} \cdot \mathbf{u}_{d}$ is the atom-field coupling constant. For simplicity we assume $g_{k}$ to be real. Here $d$ and $\mathbf{u}_{d}$ are the absolute value and the unit vector of the atomic dipole moment, $V$ is the sample volume, and $\mathbf{e}_{k}$ are the two polarization unit vectors.

Let us consider the case when the qubit is initially in its excited state $|e\rangle$ and the radiation field is in the vacuum state $|\{0\}\rangle$. The wave function of the system in the interaction picture then has the form

$$
|\psi(t)\rangle=f(t)|e\rangle|\{0\}\rangle+\sum_{k} \xi_{k}(t)|g\rangle\left|1_{k}\right\rangle
$$

where the state $\left|1_{k}\right\rangle$ accounts for the reservoir having one photon in mode $k$. The right-hand term in Eq. (2) can be written in terms of a collective state of the reservoir mode as

$$
|\psi(t)\rangle=f(t)|e\rangle|\mathbf{0}\rangle_{f}+\chi(t)|g\rangle|\mathbf{1}\rangle_{f},
$$

where the coefficient $f(t)$ is different for 1D and 3D photonic crystals. Here, we define the normalized one-photon state in the reservoir as

$$
|\mathbf{1}\rangle_{f}=\frac{1}{\chi(t)} \sum_{k} \xi_{k}(t)\left|1_{k}\right\rangle
$$

with $\chi(t)=\sqrt{\sum_{k}\left|\xi_{k}(t)\right|^{2}}=\sqrt{1-|f(t)|^{2}}$, and $|\mathbf{0}\rangle_{f}=|\{0\}\rangle$. In the following subsections, we present the calculation of $f(t)$ for the $1 \mathrm{D}$ and $3 \mathrm{D}$ photonic crystals.

\section{1D photonic crystal}

In a $1 \mathrm{D}$ photonic crystal, the dispersion characteristic of radiation waves is deformed. Near the band edge $\omega_{c}$ the dispersion relation can be approximated as

$$
\omega_{k}=\omega_{c}+A\left(k-k_{0}\right)^{2},
$$

where $A \approx \omega_{c} / k_{0}^{2}$. The amplitude $f(t)$ is given by the inverse Laplace transform

$$
f(t)=\frac{1}{2 \pi i} \int_{\sigma-i \infty}^{\sigma+i \infty} e^{s t} \tilde{f}(s) d s
$$

where $\sigma$ is a real constant that exceeds the real part of all the singularities of $\tilde{f}(s)=1 /(s+\Gamma(s))$, in which $\Gamma(s)=\frac{\gamma^{3 / 2}}{i \sqrt{-i s-\delta}}$ with $\delta=\omega_{a}-\omega_{c}$ and $\gamma^{3 / 2}=\left[\left(\omega_{a} d\right)^{2} / 6 \pi \epsilon_{0} \hbar\right]\left(k_{0}^{3} / \omega_{c}^{3 / 2}\right)$. Note that the phase angle of $\Gamma$ has been defined $-\pi / 2<\arg (\sqrt{-i s-\delta})$ $<\pi / 2$.

The amplitude $f(t)$ can be rewritten as [23]

$$
\begin{aligned}
f(t)= & \sum_{j} \frac{1}{G^{\prime}\left(x_{j}^{(1)}\right)} e^{x_{j}^{(1)} t}+\sum_{j} \frac{1}{H^{\prime}\left(x_{j}^{(2)}\right)} e^{x_{j}^{(2)} t} \\
& +\frac{e^{i \delta t} \gamma^{3 / 2}}{\pi} \int_{0}^{\infty} d x \frac{\sqrt{-i x} e^{-x t}}{i \gamma^{3}-x(-x+i \delta)^{2}}
\end{aligned}
$$

where functions $G(x)$ and $H(x)$ are defined as

$$
\begin{gathered}
G(x)=x+\frac{\gamma^{3 / 2}}{i \sqrt{-i x-\delta}}, \\
H(x)=x+\frac{\gamma^{3 / 2}}{\sqrt{i x+\delta}},
\end{gathered}
$$

$x_{j}^{(1)}$ are the pure imaginary roots of the equation $G(x)=0$ in the region $\operatorname{Im}\left(x_{j}^{(1)}\right)<\delta$ and $\operatorname{Re}\left(x_{j}^{(1)}\right)<0, x_{j}^{(2)}$ are the complex roots of the equation $H(x)=0$ in the region $\operatorname{Im}\left(x_{j}^{(2)}\right)<\delta$ and $\operatorname{Re}\left(x_{j}^{(2)}\right)<0$, and $G^{\prime}(x)=\frac{d}{d x} G(x)$ and $H^{\prime}(x)=\frac{d}{d x} H(x)$.

\section{3D photonic crystal}

In the case of $3 \mathrm{D}$ anisotropic photonic crystal, since the dispersion relation is modified strongly by the periodic dielectric structure, an anisotropic band-gap structure is formed on the surface of the first Brillouin zone in reciprocal lattice space. It is shown numerically that the band edge is associated with a finite collection of symmetry-related points $\mathbf{k}_{0}^{(\mathrm{m})}$. So, the dispersion relation can be expressed approximately by $\omega_{\mathbf{k}}=\omega_{c}+C\left|\mathbf{k}-\mathbf{k}_{\mathbf{0}}^{(\mathbf{m})}\right|^{\mathbf{2}}$, where $C$ is a model dependent constant and $\omega_{c}$ is the band edge frequency. In the same manner as $1 \mathrm{D}$ calculation, we end up having the amplitudes $f(t)$ as

$$
\begin{aligned}
f(t)= & \sum_{j} \frac{1}{A^{\prime}\left(x_{j}^{(1)}\right)} e^{x_{j}^{(1)} t}+\sum_{j} \frac{1}{B^{\prime}\left(x_{j}^{(2)}\right)} e^{x_{j}^{(2)} t}+\frac{e^{i \delta t}}{\pi} \\
& \times \int_{0}^{\infty} d x \frac{\mu^{3 / 2} i^{1 / 2} \sqrt{x}\left(\omega_{c}-i x\right) e^{-x t}}{\left[(i \delta-x)\left(\omega_{c}-i x\right)-i \sqrt{\omega_{c}} \mu^{3 / 2}\right]^{2}+i \mu^{3} x},
\end{aligned}
$$

where $\delta=\omega_{a}-\omega_{c}, \mu=\left[\frac{\epsilon_{0} \hbar C^{3 / 2}}{8 \pi}\left(\omega_{a} d\right)^{2}\left(\Sigma_{m} \sin ^{2} \theta_{m}\right)\right]^{2 / 3}$, and $\theta_{m}$ is the angle between the atomic dipole moment $\mathbf{u}_{d}$ and the $m$ th wave vector $\mathbf{k}_{0}^{(m)}$ [24]. Due to the anisotropy, the summation over $\mathbf{k}$ is replaced by an integration over $\mathbf{k}$. The integration has to be carried out around the direction of each $\mathbf{k}_{0}^{(m)}$. The function $A(x)$ and $B(x)$ are defined as

$$
\begin{aligned}
& A(x)=x-\frac{i \mu^{3 / 2}}{\sqrt{\omega_{c}}+\sqrt{-i x-\delta}}, \\
& B(x)=x-\frac{i \mu^{3 / 2}}{\sqrt{\omega_{c}}-i \sqrt{i x+\delta}},
\end{aligned}
$$

$x_{j}^{(1)}$ are the roots of $A(x)=0$ in the region $\left[\operatorname{Re}\left(x_{j}^{(1)}\right)>0\right.$ or $\left.\operatorname{Im}\left(x_{j}^{(1)}\right)>\delta\right]$, and $x_{j}^{(2)}$ are the roots of $B(x)=0$ in the region $\left[\operatorname{Re}\left(x_{j}^{(2)}\right)<0\right.$ and $\left.\operatorname{Im}\left(x_{j}^{(2)}\right)<\delta\right]$. Clearly, $x_{j}^{(1)}$ and $x_{j}^{(2)}$ depend strongly on the relative positions between $\omega$ and $\omega_{c}$, see [24,28] for more details.

In Fig. 1, we show the atomic population in the excited state for $1 \mathrm{D}$ photonic crystal as a function of time with dif- 


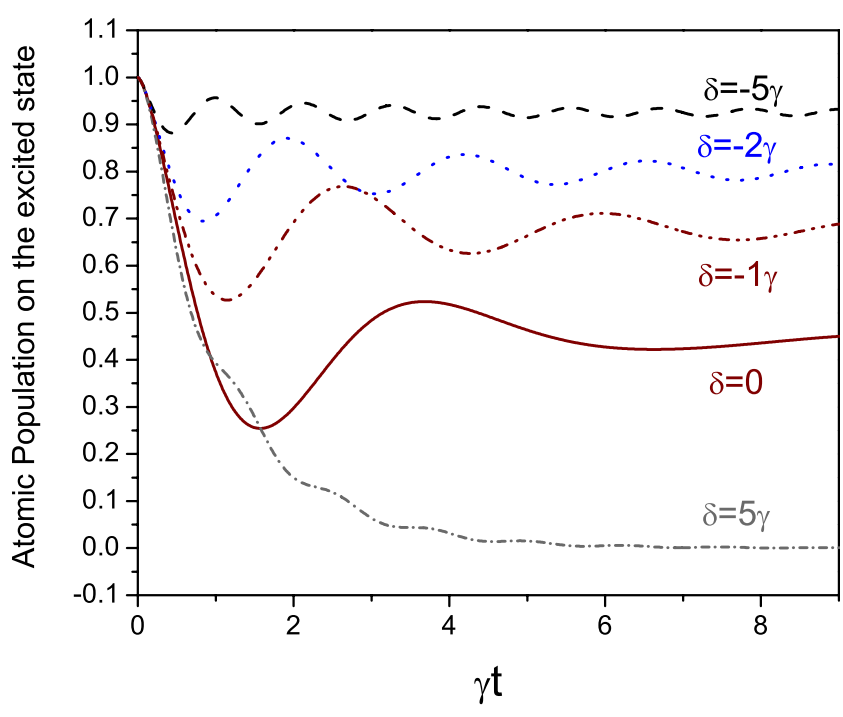

FIG. 1. (Color online) Atomic population on the excited state for 1D photonic crystal as a function of $\gamma t$; for various values of the detuning ranging from the gap to the band. Dashed curve $\delta=-5 \gamma$, dotted curve $\delta=-2 \gamma$, dashed dotted curve $\delta=-1 \gamma$, solid curve $\delta$ $=0 \gamma$, and dashed dotted curve $\delta=5 \gamma$.

ferent values of the detuning ranging from the gap to the band through the edge. The oscillatory behavior gives the indication of strong interaction between the atom and its own radiation when the atomic resonant frequency gets near the band edge. This represents the importance of the detuning as a control parameter.

In Fig. 2, we show the same but for 3D photonic crystal. The values of detuning vary from the band to the gap. It is clear that we just see fast decay of atomic population in the excited state with time. Although the oscillatory behavior can

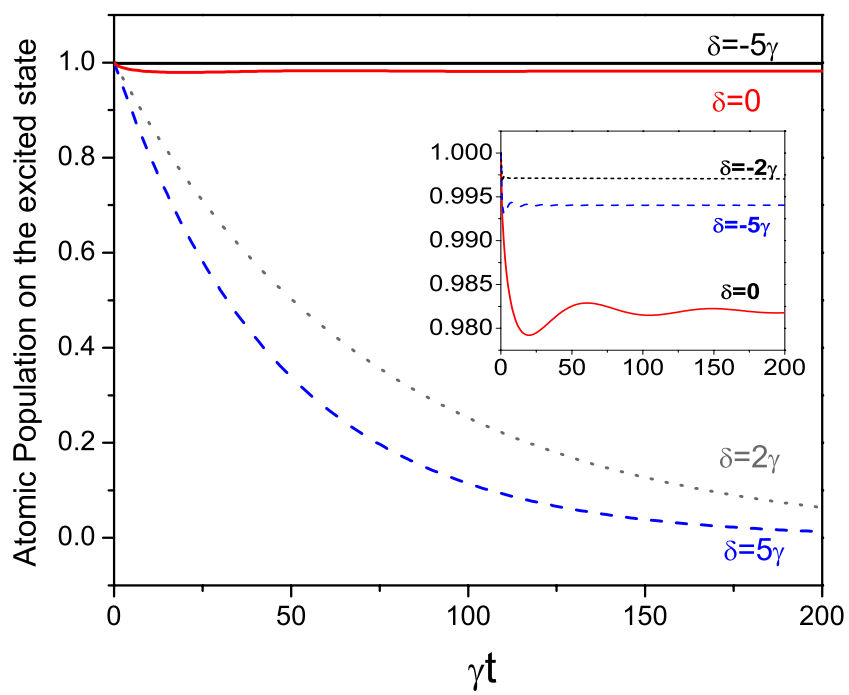

FIG. 2. (Color online) Atomic population on the excited state for $3 \mathrm{D}$ photonic crystal as a function of $\gamma t$; for various values of detuning from the band edge. black solid curve $\delta=-5 \gamma$, red solid curve $\delta=0 \gamma$, dotted curve $\delta=2 \gamma$, and dashed curve $\delta=5 \gamma$. The figure in the inset shows the same but for smaller detuning values as; $\delta=-2 \gamma$ dotted curve, $\delta=-5 \gamma$ dashed curve and $\delta=0 \gamma$ solid curve. Here $\omega_{c}=200 \gamma$. be seen in the inset where the detuning is chosen to be in the gap, this oscillation is very small and slow since the density of state is very small.

\section{B. Time evolution of the density matrix for qubits inside photonic crystals}

Here we give the time evolution of the reduced density matrix for two qubits, each of them is assumed to be placed in separate photonic crystal. Additionally, we assume that the two photonic crystals are identical and the two two-level qubits are also identical. The functions governing the dynamical behavior of each qubit have the same form as defined by $f(t)$.

We consider the initial state of the system as

$$
\rho_{a f}=\rho_{a}(0) \otimes|00\rangle\langle 00|,
$$

which means that the two qubits are initially prepared in the state $\rho_{a}(0)$ and the radiation fields in the two photonic crystals in vacuum states. For a class of the initial states that will be discussed below [29], in the representation spanned by two-qubit product states $|1\rangle=\left|e_{1}, e_{2}\right\rangle, \quad|2\rangle=\left|e_{1}, g_{2}\right\rangle, \quad|3\rangle$ $=\left|g_{1}, e_{2}\right\rangle$, and $|4\rangle=\left|g_{1}, g_{2}\right\rangle$,

$$
\rho_{a}(0)=\left(\begin{array}{cccc}
\rho_{11}(0) & 0 & 0 & \rho_{14}(0) \\
0 & \rho_{22}(0) & \rho_{23}(0) & 0 \\
0 & \rho_{32}(0) & \rho_{33}(0) & 0 \\
\rho_{41}(0) & 0 & 0 & \rho_{44}(0)
\end{array}\right) .
$$

With this initial preparation, the time-dependent reduced density matrix for the two qubits has the same structure as that of $\rho_{a}(0)$. With the time development, the nonzero matrix elements for the two qubits are listed as follows:

$$
\begin{gathered}
\rho_{11}(t)=\rho_{11}(0)\left|f_{1} f_{2}\right|^{2}, \\
\rho_{22}(t)=\rho_{11}(0)\left|f_{1}\right|^{2}\left(1-\left|f_{2}\right|^{2}\right)+\rho_{22}(0)\left|f_{1}\right|^{2}, \\
\rho_{33}(t)=\rho_{11}(0)\left|f_{2}\right|^{2}\left(1-\left|f_{1}\right|^{2}\right)+\rho_{33}(0)\left|f_{2}\right|^{2}, \\
\rho_{44}(t)=1-\rho_{11}(t)-\rho_{22}(t)-\rho_{33}(t), \\
\rho_{14}(t)=\rho_{14}(0) f_{1} f_{2}=\left[\rho_{41}(t)\right]^{*}, \\
\rho_{23}(t)=\rho_{23}(0) f_{1} f_{2}^{*}=\left[\rho_{32}(t)\right]^{*},
\end{gathered}
$$

where $f_{j}(t)(j=1,2)$ is the function characterizes the dynamics of the atom in photonic crystal as defined by Eq. (7) or (10).

\section{1. $N$ qubits initially prepared in a $G H Z$ state}

Next we consider $N$ qubits represented by $N$ identical two-level atoms embedded in $N$ identical photonic crystals. We assume that they are initially prepared in the GHZ state as

$$
|\mathrm{GHZ}\rangle=\alpha\left|g_{1}, g_{2}, \ldots, g_{N}\right\rangle+\beta\left|e_{1}, e_{2}, \ldots, e_{N}\right\rangle
$$

where $\rho_{A}(0)$ can be expressed as 


$$
\rho_{A}(0)=|\mathrm{GHZ}\rangle\langle\mathrm{GHZ}|
$$

The reduced density matrix for the $N$ qubits evolves into

$$
\begin{aligned}
\rho(t)= & |\beta|^{2} f^{2 N}\left|e_{1}, e_{2} \ldots, e_{N}\right\rangle\left\langle e_{1}, e_{2} \ldots, e_{N}\right| \\
& +\alpha^{*} \beta f^{N}\left|e_{1}, e_{2} \ldots, e_{N}\right\rangle\left\langle g_{1}, g_{2} \ldots, g_{N}\right| \\
& +\alpha \beta^{*} f^{* N}\left|g_{1}, g_{2} \ldots, g_{N}\right\rangle\left\langle e_{1}, e_{2}, \ldots, e_{N}\right| \\
& +|\beta|^{2}\left(1-|f|^{2}\right)|f|^{2(N-1)} \sum_{q=1}^{N}\left|g_{q},\{e\}_{N-1}\right\rangle\left\langle g_{q},\{e\}_{N-1}\right| \\
& +\cdots+|\beta|^{2}\left(1-|f|^{2}\right)^{2}|f|^{2(N-2)} \\
& \times \sum_{j \neq q=1}^{N}\left|g_{j}, g_{q},\{e\}_{N-2}\right\rangle\left\langle g_{j}, g_{q},\{e\}_{N-2}\right| \\
& +\cdots+\left(|\alpha|^{2}+|\beta|^{2}\left(1-f^{2}\right)^{N}\right)\left|g_{1}, g_{2} \ldots, g_{N}\right\rangle\left\langle g_{1}, g_{2} \ldots, g_{N}\right|
\end{aligned}
$$

where $\left|g_{q},\{e\}_{N-1}\right\rangle$ represents that the $q$ th qubit is in its ground state and the rest $N-1$ qubits in their excited states, and $\left|g_{j}, g_{q},\{e\}_{N-2}\right\rangle$ describes that the $j$ th and the $q$ th qubits are in their ground states and the rest $N-2$ qubits in their excited states,

\section{TIME EVOLUTION OF THE ENTANGLEMENT FOR THE QUBITS EMBEDDED SEPARATELY IN PHOTONIC CRYSTALS}

First we consider the entanglement for the two-qubit case. Later we shall consider the case for $N$ qubits.

\section{A. Sudden death and birth of entanglement between two-qubit in $1 D$ and $3 D$ crystals}

For a two-qubit system described by the density operator $\rho_{a}(t)$, a measure of entanglement can be defined in terms of the negativity [30-34] $E=\min \left(0, \Sigma \Lambda_{i-}\right)$, where $\Lambda_{i-}$ are the negative eigenvalues of the partial transposition of $\rho_{a}(t)$. The value of $E=-1 / 2$ corresponds to the maximum entanglement between the two qubits while $E=0$ describes completely separated qubits. After some algebra, the possible negative eigenvalues of the partial transposition of $\rho_{a}(t)$ in our case is given by

$$
\begin{aligned}
& \Lambda_{1-}=\frac{1}{2}\left(\rho_{11}+\rho_{44}-\sqrt{\left(\rho_{11}-\rho_{44}\right)^{2}+4 \rho_{23} \rho_{32}}\right), \\
& \Lambda_{2-}=\frac{1}{2}\left(\rho_{22}+\rho_{33}-\sqrt{\left(\rho_{22}-\rho_{33}\right)^{2}+4 \rho_{14} \rho_{41}}\right) .
\end{aligned}
$$

The above equations indicate that the appearance of the entanglement between the two qubits requires that at least one of the two inequalities $\left|\rho_{23}\right|>\sqrt{\rho_{11} \rho_{44}}$ and $\left|\rho_{14}\right|>\sqrt{\rho_{22} \rho_{33}}$ is satisfied.

In the following, we use this formalism to investigate the dynamics of entanglement between the two two-level qubits separately embedded in two photonic crystals. For the following initial state:

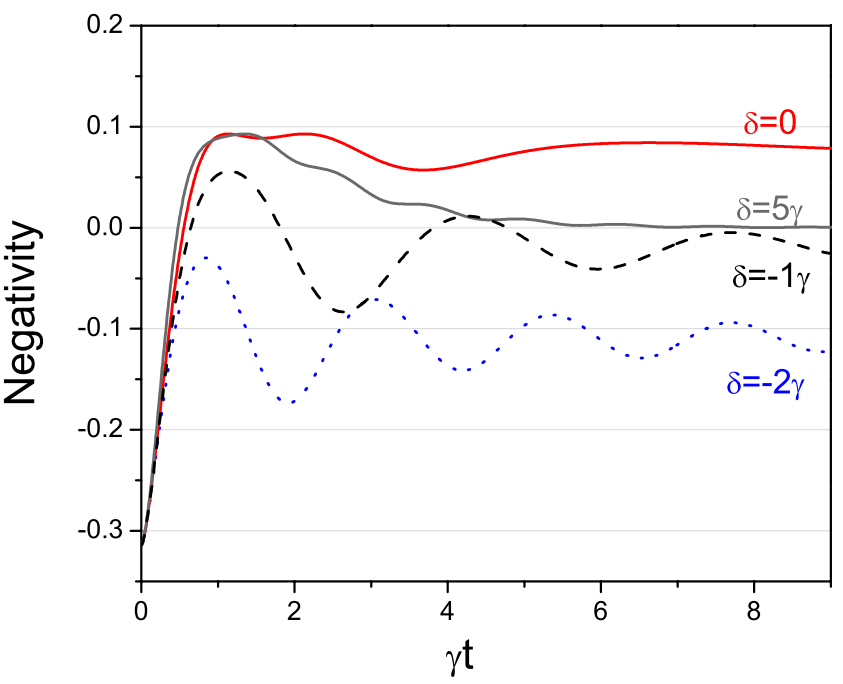

FIG. 3. (Color online) Negativity as a function of $\gamma t$ for $1 \mathrm{D}$ photonic crystal. Different values of detuning as; red solid curve $\delta=0 \gamma$, gray solid curve $\delta=5 \gamma$, dashed curve $\delta=-1 \gamma$, dotted curve $\delta=-2 \gamma$. Here, $\alpha=1 / 3$ and $\beta=\sqrt{8} / 3$.

$$
\rho(0)=\left[\beta\left|e_{1}, e_{2}\right\rangle+\alpha\left|g_{1}, g_{2}\right\rangle\right]\left[\beta\left\langle e_{1}, e_{2}\right|+\alpha\left\langle g_{1}, g_{2}\right|\right]
$$

with $\alpha$ and $\beta$ satisfying $\alpha^{2}+\beta^{2}=1$, we can see that the eigenvalue $\Lambda_{1-}$ cannot be negative and only $\Lambda_{2-}$ characterizes the time evolution of the entanglement for the two qubits. Moreover, sudden death happens when $\beta>\alpha$.

If the two qubits have the same resonant transition frequencies $\omega_{a 1}=\omega_{a 2}$ and the two photonic crystals have the same properties, then we have $f_{1}=f_{2}=f$. It is evident that, when the function $|f(t)|^{2}$ obeys

$$
|f(t)|^{2} \leq 1-\frac{\left|\rho_{14}(0)\right|}{\rho_{11}(0)}=1-\frac{\alpha}{\beta},
$$

the entanglement disappears. Evidently $|f(t)|^{2}$ is the function governing the dynamical behavior of each qubit in photonic crystal due to the spontaneous emission, and the ratio $\frac{\left|\rho_{14}(0)\right|}{\rho_{11}(0)}$ which is the initial two-photon coherence with the initial population of the two qubits in their excited states. Consequently under the influence of spontaneous emission, there may exist the sudden-death phenomenon by carefully choosing $\alpha / \beta$. The sudden death time is decided by the detuning $\delta$ and $\alpha / \beta$. However, because of the existence of the atomfield bound state in the system of qubits embedded in the photonic crystal, the function $|f(t)|^{2}$ can do a damped Rabi oscillation as shown in Figs. 1 and 2. This can lead to the entanglement birth. The reason for the entanglement birth can be understood as follows. The initially entangled qubits spontaneously emit the photons into the photonic crystals which may cause the two qubits become unentangled. However, due to the existence of the atom-field bound state, the qubits can absorb these photons again and the initial entanglement between the qubits is partially recovered. A close look at Fig. 3 shows some of these interesting features for the case of $1 \mathrm{D}$ crystals. For the detuning at the edge $\delta=0$ we have the entanglement disappearing after some time. The interesting phenomena take place when the detuning is cho- 


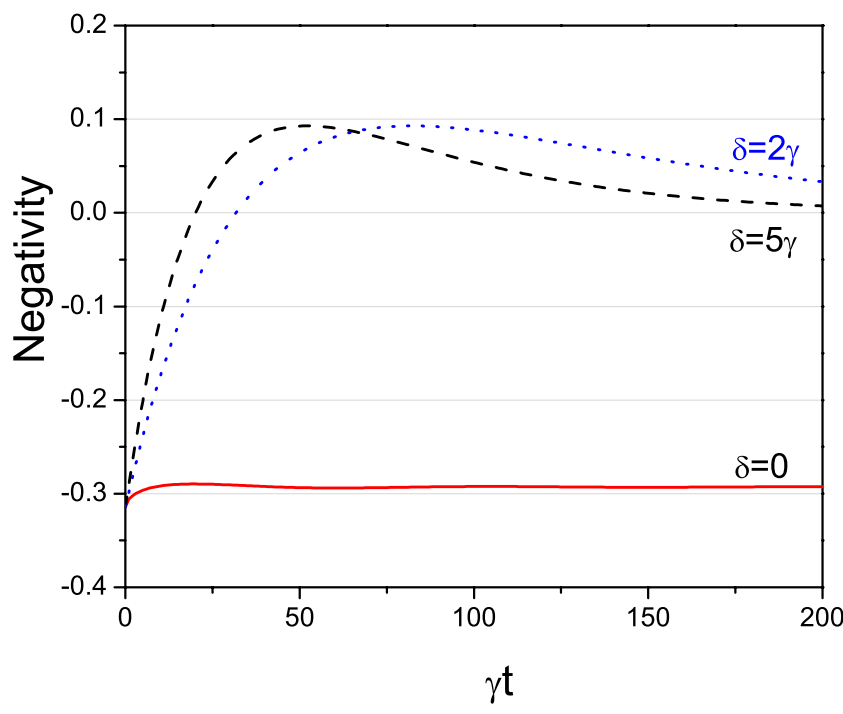

FIG. 4. (Color online) Negativity as a function of $\gamma t$ for 3D photonic crystal. Different values of detuning as; dotted $\delta=2 \gamma$, dashed curve $\delta=5 \gamma$, solid curve $\delta=0 \gamma$. Here, $\omega_{c}=200 \gamma, \alpha=1 / 3$, and $\beta=\sqrt{8} / 3$.

sen to be $\delta=-1 \gamma$. Here the entanglement has sudden death for some time and then we have sudden birth. The entanglement can then survive in the long time limit. If the transition frequency is deep in the gap then the entanglement is preserved as in the case of $\delta=-2 \gamma$. Another point to note is that, when the transition frequency is deeper in the band, we have sudden death of entanglement. The deeper the transition frequency in the band, the faster the sudden death is as, for example, in the case when the detuning $\delta=5 \gamma$.

In the long time limit, the population function for each qubit in its excited state $|e\rangle$ is reduced as

$$
|f(\infty)|^{2}=\left|2 z^{3} /\left(1+2 z^{3}\right)\right|^{2},
$$

in which $z=(1 / 2+\Xi)^{1 / 3}+(1 / 2-\Xi)^{1 / 3}$ with $\Xi$ $=\sqrt{1 / 4+\left(\omega_{a c} / 3 \gamma\right)^{3}}$. This is due to the birth of the atom-field bound state, which results in the slow decay of the qubits. Therefore, the entanglement between the two qubits can survive, see Fig. 3.

The case of 3D photonic crystal represents a different scenario as shown in Fig. 4. It is clear that the entanglement is maintained once the transition frequency is at the edge or inside the gap. The reason is that the density of state is very small in these cases. However, if the transition frequency happens to be in the band region at $\delta=2 \gamma, 5 \gamma$, then the entanglement disappears in fast decay manner. Generally speaking, entanglement can be preserved longer if the transition frequency of the qubit gets closer to the edge from the band region. Again, it is evident that the qubit dynamical behavior is restricted by the initial coherence and the population in the excited state.

\section{B. Sudden death of entanglement among $N$ qubits in 1D and 3D crystals}

Now we turn our attention to discuss the dynamical behavior of the entanglement among $N$ qubits, each of them is, respectively, embedded in one photonic crystal. Similar to the two qubits case, here we adopt the negativity, which is defined as the sum of the negative eigenvalues of the partially transposed density matrix as a quantifier of the entanglement. In general, the negativity cannot always characterize the entanglement property of the entangled states in dimension higher than six. However, for the state as described by Eq. (18), if we treat the first $q$ qubits as a partition and the rest $N-q$ as another one, as shown in the following, the submatrix for the partial transposition reduces to a fourdimension problem. So the null negativity means separability in the corresponding partition. When we treat the first $q$ qubits as a partition and $N-q$ as another one, the submatrix for the partial transposition is

$$
\begin{aligned}
\rho^{T}= & \lambda_{q}\left|g_{1}, \ldots, g_{q}, e_{q+1} \ldots, e_{N}\right\rangle\left\langle g_{1}, \ldots, g_{q}, e_{q+1} \ldots, e_{N}\right| \\
& +\lambda_{N-q}\left|e_{1}, \ldots, e_{q}, g_{q+1} \ldots, g_{N}\right\rangle\left\langle e_{1}, \ldots, e_{q}, g_{q+1} \ldots, g_{N}\right| \\
& +\alpha \beta^{*} f^{* N}\left|e_{1}, \ldots, e_{q}, g_{q+1} \ldots, g_{N}\right\rangle\left\langle g_{1}, \ldots, g_{q}, e_{q+1} \ldots, e_{N}\right| \\
& +\alpha^{*} \beta f^{* N}\left|g_{1}, \ldots, g_{q}, e_{q+1} \ldots, e_{N}\right\rangle\left\langle e_{1}, \ldots, e_{q}, g_{q+1} \ldots, g_{N}\right|
\end{aligned}
$$

and the negativity $\Lambda$ can be represented neatly as

$$
\Lambda=\eta_{q}-\sqrt{\eta_{q}^{2}-\Delta_{q}}
$$

whereas $\eta_{q}=\left(\lambda_{q}+\lambda_{N-q}\right) / 2, \Delta_{q}=\lambda_{q} \lambda_{N-q}-|\alpha \beta|^{2}|f|^{2 N}$. The parameter $\lambda_{q}=|\beta|^{2}\left(1-|f|^{2}\right)^{q}|f|^{2(N-q)}$ is the population for $q$ qubits in their ground state. $\lambda_{N-q}=|\beta|^{2}\left(1-|f|^{2}\right)^{N-q}|f|^{2 q}$ is the population for $N-q$ qubits in the ground state. For the disappearance of entanglement we just need,

$$
|f|^{2}<1-\left(|\alpha \beta| /|\beta|^{2}\right)^{2 / N}=1-\left[\rho_{e g}(0) / \rho_{e e}(0)\right]^{2 / N},
$$

whereas $\rho_{e e}(0)$ is the initial population for all qubits in the excited states, and $\rho_{e g}(0)$ is the initial coherence between the states of all the $N$ qubits in their excited state and in their ground state. $|f(t)|^{2}$ is governing the dynamical behavior of each qubit due to the spontaneous emission. Evidently for the two-qubit case, i.e., $N=2$, the above inequality reduces to the inequality (22). Clearly from Eq. (25) that the value of the negativity depends on the number $q$ of the qubits encoded in the first partition, but the critical time points for the sudden death or the sudden birth are independent of the parameter $q$.

Figure 5 shows the entanglement of $N=4$ qubits that separately embedded in four $1 \mathrm{D}$ crystals, respectively. Here, we have two different partitions; one in which one qubit $(q=1)$ belong to the first partition and the rest $(N-q=3)$ to the second partition. The other choice is that two atoms are treated as one partition $(q=2)$ and the rest $(N-q=2)$ belong to another one. Clearly there is a difference between the case when we have one qubit as one partition and the case of two qubits as another system. However, the sudden death and sudden birth time happen at the same time for both partitions. The inset shows the same but for $N=5$ qubits. In both figures, we can see that the entanglement is preserved for the same parameters.

Figure 6, and the inset there in, shows the entanglement of $N=4$ and $N=5$ qubits that separately embedded in four and five $3 \mathrm{D}$ crystals, respectively. Again one can easily notice 


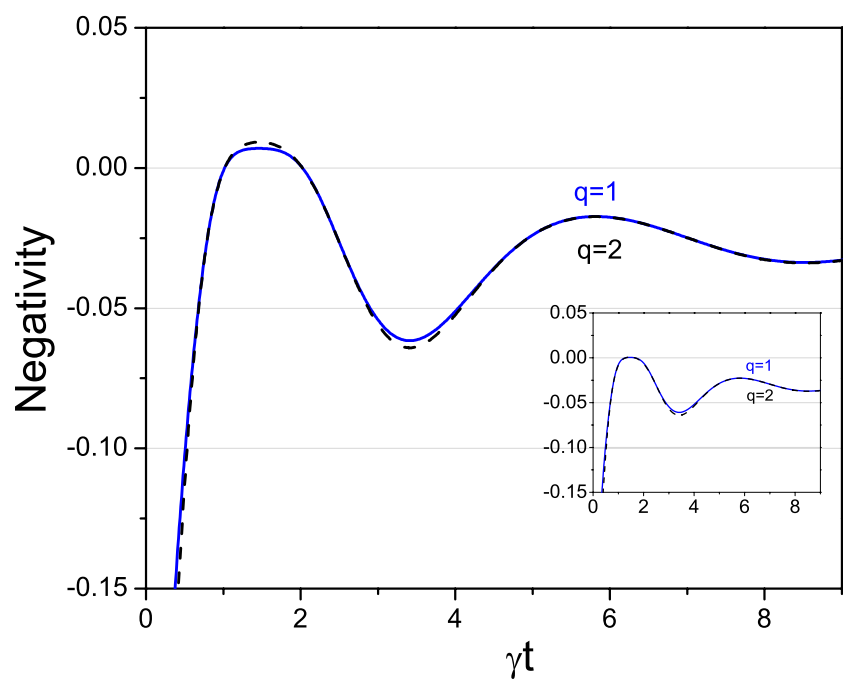

FIG. 5. (Color online) Negativity as a function of $\gamma t$ for 1D photonic crystal. Here, detuning $\delta=-0.25 \gamma, N=4$, solid curve $q$ $=2$, dotted curve $q=1, \alpha=1 / 3$, and $\beta=\sqrt{8} / 3$. The inset represent the same but for $N=5$.

that there is a difference between the case when we have one qubit as one partition and the case of two qubits as another one. The general trend in the $3 \mathrm{D}$ photonic crystal is the fast decay of the entanglement.

Figure 7, we plot the negativity of 1D crystal for different $N$ qubits and just considering one partition when $q=1$. Clearly, the sudden birth and sudden death of entanglement depends on the number of the qubits in the system. For small number of qubits we can see sudden death and sudden birth very clearly as in the case of $N=3$. Once we increase the number of qubits to $N=5$, we just see death of the entanglement and one birth. Moreover, for large number of qubits $N=50$, it is very clear that entanglement just decays fast with time.

Figure 8 gives clear indication that the entanglement death is preserved no matter how many qubits we have in the

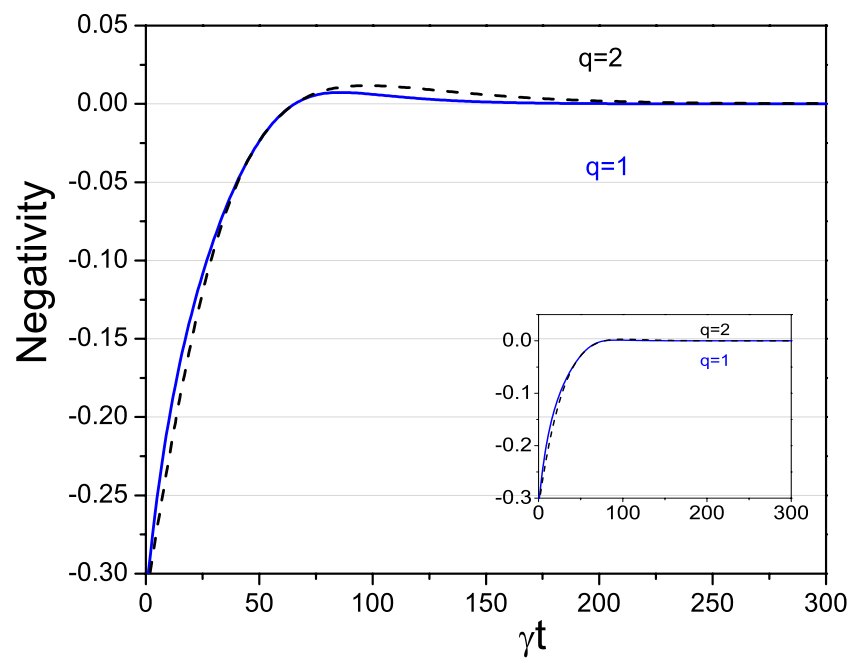

FIG. 6. (Color online) Negativity as a function of $\gamma t$ for 3D photonic crystal. Here, $\omega_{c}=200 \gamma, \delta=2 \gamma, N=4$, solid curve $q=1$, dotted curve $q=2, \alpha=1 / 3$ and $\beta=\sqrt{8} / 3$. The inset represent the same but for $N=5$.

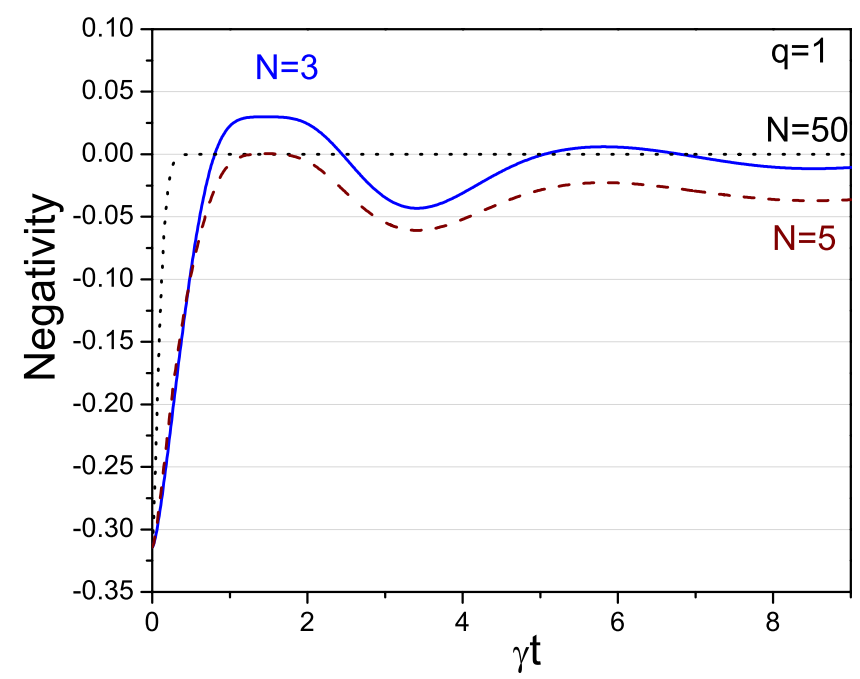

FIG. 7. (Color online) Negativity as a function of $\gamma t$ for 1D photonic crystal. For different values of $N$, solid curve $N=3$, dashed curve $N=5$, and dotted curve $N=50$. Here, $q=1$, detuning $\delta=$ $-0.25 \gamma, \alpha=1 / 3$, and $\beta=\sqrt{8} / 3$.

system. However, this death depends on the number of the qubits in the crystal. Clearly the time of entanglement death is different from one case to another.

From the above discussion we can conclude that, for a multiqubit system initially in the GHZ state, entanglement death is observed in the $3 \mathrm{D}$ photonic crystal if the transition frequency of each qubit is located in the band. The entanglement, however, can be well preserved in the 3D photonic crystal once the transition frequency is inside the gap. The scenario is somewhat different in the 1D photonic crystal since someone can see entanglement sudden death and birth. That is due to the existence of atom-photon bound state. Even if the transition frequency of each qubit lies in the gap, the partial entanglement will be lost even in the disappearance of the sudden death of entanglement. This is due to the

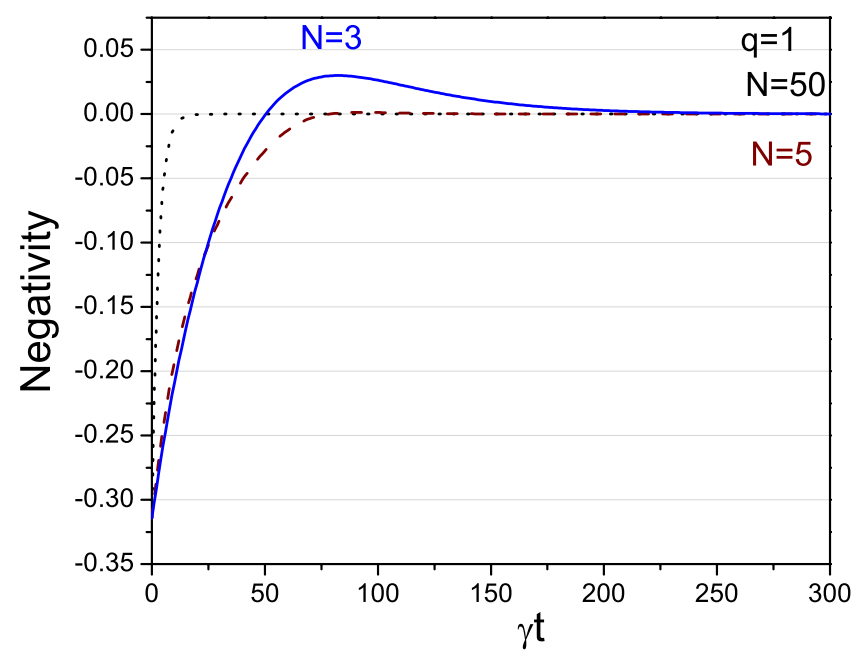

FIG. 8. (Color online) Negativity as a function of $\gamma t$ for 3D photonic crystal. For different values of $N$, solid curve $N=3$, dashed curve $N=5$, and dotted curve $N=50$. Here, $q=1, \omega_{c}=200 \gamma, \delta=2 \gamma$, $\alpha=1 / 3$ and $\beta=\sqrt{8} / 3$. 
difference between the DOS in 3D photonic crystal and that in 1D photonic crystal. Therefore, to store the entanglement for multi qubits system initially in the GHZ state, embedding each qubit separately in $3 \mathrm{D}$ photonic crystal is much better than in 1D photonic crystal.

\section{CONCLUSION}

In conclusion, we have shown that sudden death and sudden birth of entanglement do appear in the case of two qubits placed in two 1D photonic crystals. The sudden death time of entanglement is decided by the detuning $\delta$, the initial coherence, the population in the excited state and $\alpha / \beta$. Due to the existence of the atom-field bound state the function $|f(t)|^{2}$ can do a damped Rabi oscillation, which causes the birth of the entanglement. In the case of 3D photonic crystals, we just see sudden death and no birth since the qubit dynamical behavior is restricted by the initial coherence and the population in the excited state which results in fast decay of the entanglement. However, entanglement can be preserved longer if the transition frequency of the qubit gets closer to the edge from the band and gap regions.

We also have presented a neat expression for the negativity when we have $N$ qubits in $N$ photonic crystals. The value of the negativity depends on the number $q$ of the qubits encoded in the first partition, but the critical time points for the sudden death or the sudden birth are independent of the parameter $q$.

\section{ACKNOWLEDGMENTS}

This research is supported by grants from the King Abdul Aziz City of Science and Technology (KACST) and the Qatar National Research Fund (QNRF). M.S.Z. would also like to thank the Alexander von Humboldt Foundation for supporting this research. G.X.L. would like to thank the financial support from the National Natural Science Foundation of China (under Grants No. 10674052 and No. 60878004) and the Ministry of Education under project NCET (under Grant No. NCET-06-0671).
[1] L. Diósi, Lect. Notes Phys. 622, 157 (2003).

[2] A. Barenco, Contemp. Phys. 37, 375 (1996).

[3] M. A. Nielsen and I. L. Chuang, Quantum Computation and Quantum Information (Cambridge University Press, Cambridge, England, 2000).

[4] T. Yu and J. H. Eberly, Phys. Rev. Lett. 93, 140404 (2004).

[5] T. Yu and J. H. Eberly, Phys. Rev. Lett. 97, 140403 (2006).

[6] J. H. Eberly and T. Yu, Science 316, 555 (2007).

[7] A. Al-Qasimi and D. F. V. James, Phys. Rev. A 77, 012117 (2008).

[8] L. Derkacz and L. Jakóbczyk, Phys. Rev. A 74, 032313 (2006).

[9] M. O. T. Cunha, New J. Phys. 9, 237 (2007).

[10] S. Natali and Z. Ficek, Phys. Rev. A 75, 042307 (2007).

[11] M. P. Almeida, F. De Melo, M. Hor-Meyll, A. Salles, S. P. Walborn, P. H. S. Ribeiro, and L. Davidovich, Science 316, 579 (2007).

[12] M. F. Santos, P. Milman, L. Davidovich, and N. Zagury, Phys. Rev. A 73, 040305(R) (2006).

[13] Z. Ficek and R. Tanaś, Phys. Rev. A 74, 024304 (2006).

[14] Z. Ficek and R. Tanaś, Phys. Rev. A 77, 054301 (2008).

[15] C. E. López, G. Romero, F. Lastra, E. Solano, and J. C. Retamal, Phys. Rev. Lett. 101, 080503 (2008).

[16] Z. Zhao, Y. A. Chen, A. N. Zhang, T. Yang, H. J. Briegel, and J. W. Pan, Nature (London) 430, 54 (2004).

[17] W. B. Gao, C. Y. Lu, X. C. Yao, P. Xu, O. Gühne, A. Goebel, Y. A. Chen, C. Z. Peng, Z. B. Chen, and J. W. Pan, e-print arXiv:0809.4277.

[18] H. Häffner et al., Nature (London) 438, 643 (2005).

[19] W. Dür and H. J. Briegel, Phys. Rev. Lett. 92, 180403 (2004).
[20] L. Aolita, R. Chaves, D. Cavalcanti, A. Acín, and L. Davidovich, Phys. Rev. Lett. 100, 080501 (2008); L. Aolita, D. Cavalcanti, A. Acín, A. Salles, M. Tiersch, A. Buchleitner, and F. de Melo, Phys. Rev. A 79, 032322 (2009).

[21] F.-Q. Wang, Z.-M. Zhang, and R.-S. Liang, Phys. Rev. A 78, 042320 (2008).

[22] B. Bellomo, R. Lo Franco, S. Maniscalco, and G. Compagno, Phys. Rev. A 78, 060302(R) (2008).

[23] Y. Yang and S.-Y. Zhu, Phys. Rev. A 61, 043809 (2000); G. X. Li, F. L. Li, and S. Y. Zhu, ibid. 64, 013819 (2001).

[24] Y. Yang and S.-Y. Zhu, Phys. Rev. A 62, 013805 (2000); S. Y. Zhu, G. X. Li, Y. P. Yang, and F. L. Li, Europhys. Lett. 62, 210 (2003).

[25] D. G. Angelakis, P. L. Knight, and E. Paspalakis, Contemp. Phys. 45, 303 (2004).

[26] S. John and T. Quang, Phys. Rev. A 50, 1764 (1994).

[27] M. O. Scully and M. S. Zubairy, Quantum Optics (Cambridge University Press, New York, 1997).

[28] S.-Y. Zhu, Y. Yang, H. Chen, H. Zheng, and M. S. Zubairy, Phys. Rev. Lett. 84, 2136 (2000).

[29] M. Ikram, G. X. Li, and M. S. Zubairy, Phys. Rev. A 76, 042317 (2007).

[30] M. Horodecki, P. Horodecki, and R. Horodecki, Phys. Lett. A 223, 1 (1996).

[31] D. Kaszlikowski and A. Kay, New J. Phys. 10, 053026 (2008).

[32] G. Vidal and R. F. Werner, Phys. Rev. A 65, 032314 (2002).

[33] K. Źyczkowski, P. Horodecki, A. Sanpera, and M. Lewenstein, Phys. Rev. A 58, 883 (1998).

[34] A. Peres, Phys. Rev. Lett. 77, 1413 (1996). 